\title{
Atravesando la ciudad. La movilidad y experiencia subjetiva del espacio por las empleadas domésticas en Bogotá
}

Friederike Fleischer. Universidad de Los Andes, Bogotá, Colombia. Keren Marín. Universidad de Los Andes, Bogotá, Colombia.

RESUMEN | Enfocado en las vidas y experiencias de las empleadas domésticas de Bogotá, Colombia, este artículo examina la interrelación entre el espacio y las diferencias socioeconómicas que demarcan el contexto urbano. De manera específica, se explora las formas en que las empleadas domésticas "producen el espacio" (Lefebvre, 1991). Para ello se analiza, a través de entrevistas no-estructuradas y la documentación de las trayectorias de movilidad, la forma en que estas mujeres usan, experimentan e intervienen el espacio urbano. El artículo se propone, en suma, ofrecer elementos para comprender la manera en que las lógicas de estratificación de la ciudad enmarcan la experiencia subjetiva de las empleadas domésticas. En este sentido, el análisis se enfoca en las interacciones de las dimensiones espaciales, sociales y económicas, y en cómo estas definen las vidas y posibilidades de movilidad de las empleadas domésticas en la ciudad.

PALABRAS CLAVE | desigualdad social, segregación, movilidad.

ABSTRACT | Domestic workers are a vital element of Latin American urban society. Focusing on the lives and experiences of household employees in Bogotá, Colombia, this article examines the interrelation between space and socioeconomic differences in the urban context. More specifically, we explore female domestic workers' "production of space" (Lefebvre, 1991), specifically analyzing how they use, experience, and intervene in the urban space. By following household employees in their trajectories, the project illuminates how space is stratified, how the women experience the space, and how spatial, social, and economic dimensions interact with each other to impact their lives and mobility possibilities. The research shows how spatial and socioeconomic mobility is put into practice and negotiated daily, not only in the city itself but also through extensions of time and space beyond the urban sphere.

KEYWORDS | social inequality, segregation, mobility. 


\section{Introducción}

Al igual que en otras ciudades latinoamericanas, la estratificación de Bogotá no solo se expresa en términos socioeconómicos, sino también de manera espacial. De hecho, la estratificación está inscrita en las políticas urbanas y de planeación: la ciudad está organizada según las características socioeconómicas del espacio, y con base en tal parámetro es clasificada en una escala de uno (estrato 1, bajo) a seis (estrato 6, alto), considerando la relación vivienda-entorno (figura 1). Esta clasificación es la base para calcular el impuesto predial y los costos de los servicios públicos domiciliarios por medio de tarifas diferenciales, donde los estratos altos pagan proporcionalmente más que los bajos (Uribe \& Pardo, 2006). Este tipo de estratificación urbana hace parte del sentido común de la gente en Colombia y no solamente permite simplificar las realidades socioeconómicas urbanas, ${ }^{1}$ sino que contribuye a la inscripción de la estratificación en términos geográficos y sociales. De hecho, el sistema de estratos crea diversos imaginarios urbanos sobre la ciudad y los sujetos que la habitan. En este sentido, la estratificación sirve como descripción de la apariencia del paisaje, seguridad del entorno y calidad de los servicios de cada área en la ciudad y es un medio a través del cual se representan sus habitantes. Así pues, alguien es "estrato 1" cuando carece de ciertas cualidades asociadas con la clase media y alta, cuyos integrantes son clasificados como "gente con estrato". Además, ser residente de ciertas localidades de la ciudad se asocia, usualmente, con la pertenencia a cierta clase socioeconómica. Este tipo de imaginarios no solo estigmatiza a parte de la población urbana, sino que además impide el acceso a la ciudad de manera equitativa y justa, pues frente al miedo al "otro" la ciudad sufre un proceso de segregación espacial (Hiernaux, 2007; Lindón, 2006). ${ }^{2}$

Un contexto urbano de esta naturaleza, donde las diferencias socioeconómicas definen no solo políticas públicas sino también aspectos de la subjetividad de los sujetos -en particular su representación identitaria-, afecta las condiciones que enmarcan el trabajo doméstico, pues este se encuentra atado a las diferencias socioeconómicas del país. En este sentido, las empleadas domésticas de acuerdo con los resultados de nuestra investigación, pertenecen usualmente a las clases bajas (estratos 1 y 2) y trabajan para las clases más altas (estratos 4 a 6). Nuestro trabajo expone cómo esta diferencia se expresa de manera espacial, pues el viaje diario que realizan las empleadas atraviesa toda la ciudad: desde el sur, en los barrios marginales donde habitan, hasta las casas de sus empleadores, que suelen estar ubicadas en el norte de Bogotá. El recorrido implica salir de barrios más heterogéneos en términos de clase, etnia y origen social, hacia sectores más ricos y homogéneos socialmente, espacios

1 Existe, por ejemplo, la pobreza escondida dentro de barrios asignados con un estrato medio-alto (Téllez Oliveros, 2014).

2 Al respecto, véase "Los estratos 'acentúan la segregación y los estereotipos': UnAL", reseña de investigación de la Universidad Nacional de Colombia (UNAL) y la Secretaría Distrital de Planeación (SDP), en revista Dinero (1/14/2016), https://bit.ly/2xU7Fae. Resultados de la investigación en F. Bogliacino, L. Jiménez y D. Reyes, "Identificar la incidencia de la estratificación socioeconómica urbana sobre la segregación de los hogares bogotanos", Investigaciones y Productos CID [Centro de Investigaciones para el Desarrollo, Universidad Nacional de Colombia], 24 (diciembre 2015), https://bit.ly/22kTuUF 
en donde ellas están corporalmente marcadas como "el otro". Aquí, las empleadas domésticas entran a la esfera privada de sus empleadores y de esta manera cruzan también divisiones conceptuales de barrio, género y privacidad.

FIGURA I | Estratificación socioeconómica de Bogotá D.C.

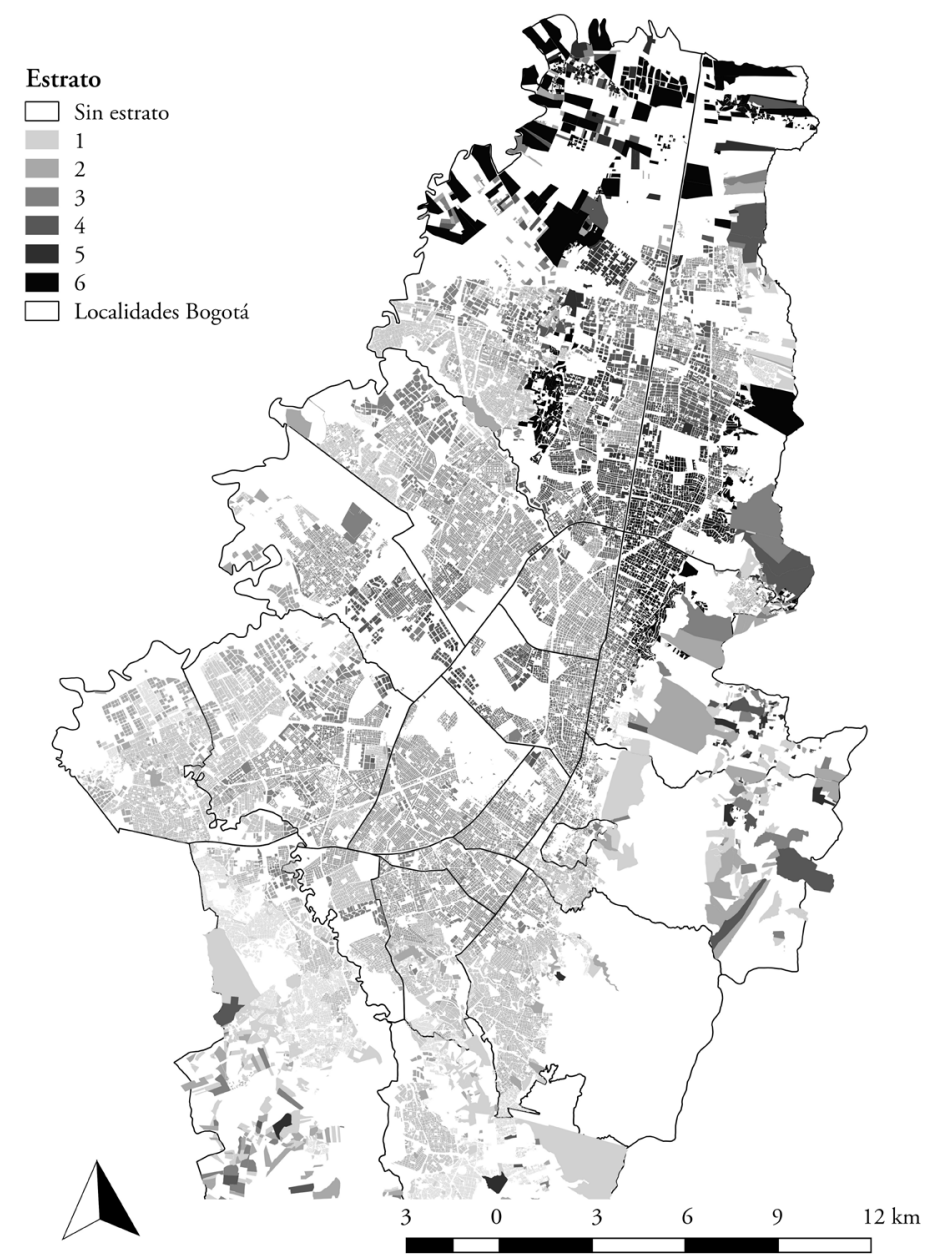

FUENTE UNIDAD ADMINISTRATIVA ESPECIAL DE CATASTRO DISTRITAL, 2017

Enfocado en las vidas y experiencias de las empleadas domésticas de Bogotá, este artículo explora cómo las dimensiones sociales y económicas del trabajo doméstico se interrelacionan con la espacialidad. Por consiguiente, analizaremos "la producción del espacio" (Lefebvre, 1991) por parte de las empleadas domésticas y de qué manera la interacción de esta población con el ámbito urbano moldea las ciudades en Colombia hoy en día. El texto expone cómo el espacio está estratificado, qué experiencia de este tienen las mujeres y la manera en que las dimensiones espaciales, 
sociales y económicas interactúan para impactar sus vidas y sus posibilidades de movilidad. En conclusión, el texto ilumina las formas, experiencias, posibilidades y límites de la movilidad social y espacial como un fenómeno específico de lugar y tiempo.

El artículo se inicia explicando la metodología de esta investigación, a lo que sigue una exposición de las distintas perspectivas analíticas para comprender el espacio, el empleo doméstico, así como los puntos de encuentro entre estos temas. En la tercera parte introducimos a dos de nuestras interlocutoras, los barrios en que viven y sus desafíos de movilidad en relación con sus labores como empleadas domésticas. En ese apartado analizamos cómo se interrelacionan las dimensiones espaciales, económicas y sociales con la vida diaria y las experiencias subjetivas de estas mujeres en términos geográficos. La última sección del texto ofrece algunas conclusiones y recomendaciones generales a partir de las observaciones realizadas en la ciudad y los testimonios de varias de nuestras interlocutoras.

\section{Metodología de investigación}

Esta investigación, al inscribirse en el campo de la antropología urbana, se desarrolló desde una aproximación cualitativa y de corte etnográfico, enfoque que nos permitió describir y comprender las realidades y experiencias subjetivas de las empleadas domésticas a partir de las formas de interconexión y relación entre distintos fenómenos sociales, tales como la desigualdad económica, la segregación espacial o la capacidad de movilidad y movimiento. En este sentido, entre los métodos principales para la recolección de datos estuvieron la observación participante y las entrevistas no estructuradas. Esta ruta metodológica nos permitió conocer el trasfondo sociopolítico y cultural en el cual se inscriben las experiencias de vida de estas mujeres, y a la vez establecer una relación de confianza que nos permitiera acceder a otros espacios de su cotidianidad.

A partir de estas conversaciones, acompañamos a algunas de las empleadas en sus trayectorias diarias por la ciudad, aproximación que nos posibilitó analizar las formas en que interactúan con el espacio urbano, ya que durante los recorridos señalaban aquellos puntos geográficos que representan -desde su punto de vistaespacios de inseguridad, discriminación y exclusión. Esta estrategia metodológica estuvo acompañada de un proceso de georreferenciación y mapeo espacial, el cual nos permitió dimensionar la capacidad de motilidad de las mujeres en el entorno urbano. A través de esta ruta metodológica logramos entrevistar a veintisiete mujeres de distintos grupos etarios, étnicos y socioeconómicos, muestra que, al ser plural, resulta representativa para comprender las realidades espaciales y laborales de las empleadas domésticas en Bogotá.

\section{Segregación socioespacial, movilidad y empleo doméstico. Un balance conceptual}

Entre las principales corrientes del estudio de las lógicas urbanas, la geografía crítica y la geografía marxista realizaron las primeras contribuciones relativas a las formas de producción social del espacio. En este sentido, estas corrientes se han centrado en 
analizar los procesos de segregación espacial en las ciudades, es decir, la distribución desigual de grupos sociales en el espacio físico (Järv, 2014). Al respecto, David Harvey (1977) y Neil Smith (2000) mostraron cómo el mercado del suelo (en particular el "segundo circuito del capital") en el sistema capitalista genera segregación, exclusión y marginalización en la ciudad.

En Latinoamérica, según Torres Tovar (2002), la alta y rápida tasa de urbanización ha contribuido al "aumento de las áreas periféricas de urbanización clandestina, invasiones de terrenos públicos, traslado a urbanizaciones de moda en lugares alejados de la ciudad por parte de las familias de ingresos medios y altos, y un deterioro de las áreas centrales antiguas y proliferación de inquilinatos. Desigualdad, desempleo, miseria urbana, alta demanda de servicios públicos y equipamientos colectivos, desorden urbanístico y extensión antieconómica de las principales ciudades, a costa de la ocupación de tierras de alto potencial agrícola, entre otros factores" (p. 319). Para el caso específico de Bogotá, Fuentes Durán (2010) argumenta que la segregación socioespacial está arraigada en la evolución y determinación del precio del suelo y que el mecanismo siempre perjudica a la población urbana más desfavorecida.

Los procesos de segregación espacial también han sido comprendidos como resultado de imaginarios morales y cognitivos sobre la otredad y su entorno. Ejemplo de ello es el caso de los barrios mixtos en Santiago de Chile, donde a pesar de la proximidad física y espacial entre clases medias y populares, prima la desconfianza y el deseo de distancia social (Ruiz, 2016; Santillán, 2015; Saraví, 2008). Ante ello, la ciudad es planeada con base en el deseo de excluir, mas no integrar, lo cual ha tenido como consecuencias la construcción de barrios en las periferias, la existencia de una ciudad privada y cerrada para las clases populares y la inadecuación entre la localización de los empleos, los servicios y los lugares de residencia (Kaztman, 2001; Soja, 2008).

Estas perspectivas analíticas -centradas en el estudio de la materialidad y las relaciones de poder en la trama urbana- se ampliaron en la década de los noventa a partir del giro de la movilidad, un enfoque incorporado a la geografía urbana que permitió entender las dinámicas de la urbe en términos de representaciones, apropiaciones y corporeidades. Sheller y Urry $(2006,2016)$ propusieron el "nuevo paradigma de la movilidad", el cual resaltó el rol constitutivo de los movimientos para las instituciones y prácticas sociales. Este paradigma examina lo corporal, virtual e imaginado en los viajes de personas, objetos e ideas. Además, enfatiza la relación entre movilidad e infraestructura, punto importante para la presente investigación. En este sentido, se empezaron a comprender los procesos de movilidad en términos de redes, relaciones, flujos y como fenómenos heterogéneos, influenciados por factores socioculturales como la edad, ocupación y nivel económico, entre otros (Hanson, 2010; Silvey, 2006). Ejemplo de ello son las investigaciones sobre las espacialidades imaginadas, las cuales demuestran que la percepción y apropiación de la ciudad por parte de las mujeres está condicionada por el miedo y sus efectos espaciales. Así pues, el temor a la violencia sexual en espacios públicos y al acoso en los sistemas de transporte las ubica en un lugar marginal, que hace problemático ser y estar en tales espacios. En este sentido, el género es un elemento determinante 
en los procesos de accesibilidad y movilidad urbana, pues en la codificación del espacio se reproduce la idea de lo femenino y masculino, es decir, la interpretación simbólica de lo público y lo privado en la trama geográfica (Delgado, 2007; Jirón, 2007; Soto, 2017).

También podemos encontrar aproximaciones que analizan cómo la ciudad es construida, reconstruida y alterada por las trayectorias de los individuos y sus experiencias geográficas. En este sentido, la corporalidad de los sujetos resulta relevante, pues a través de ella ejecutan prácticas y recrean, mediante sus recorridos, los espacios y lugares (Lindón, 2000; Soto, 2013). Por consiguiente, el análisis del transporte resulta estratégico para examinar las vivencias en las ciudades, ya que a través de él se proporciona acceso a distintos lugares y la posibilidad de apropiación del espacio público. Esta corriente de interpretación analiza los espacios urbanos más allá de su materialidad, pues considera los procesos simbólicos, históricos y afectivos que se despliegan a partir de la experiencia espacial individual y colectiva (Bailly, 1989; Espinoza, 2013; Uribe \& Pardo, 2006).

En esta misma línea, han surgido investigaciones relacionadas con la movilidad cotidiana y su impacto en la calidad de vida. La geografía urbana se ha centrado en las experiencias y los procesos sociales relacionados con la movilidad y la facilidad de acceso a los hogares, lugares de trabajo y zonas de recreación y ocio. Desde tal perspectiva se analiza el potencial de movilidad (motility), la movilidad habitual territorial (recorridos basados en la rutina y repetición), la movilidad obligada (desplazamiento ocupacional), la movilidad no obligada (desplazamiento con fines de ocio) y las trayectorias entre los espacios de actividad personal, los de residencia y los espacios cotidianos (Ares, 2010; Urry, 2011). La movilidad aparece, entonces, como una nueva forma de capital, pues la posibilidad de movilizarse espacialmente depende en gran medida del acceso a recursos económicos: la ciudad es, así, el lugar donde el poder se refuerza y refleja (Dureau, 2013; Jirón, 2007; Kaufmann, Bergman \& Joye, 2004; Roca, 2012; Thibert \& Osorio, 2014; Uribe \& Pardo, 2006).

No obstante, y a pesar de las múltiples perspectivas y enfoques en la comprensión de la ciudad y sus dinámicas, los estudios relacionados con empleo doméstico y movilidad son mínimos. En Latinoamérica, los trabajos de Elizabeth Jelin (1977), Magdalena León (1982), Alda Da Britto Motta (1985), Elsa Chaney y Mary García (1989), Suely Kofes (1990) y Christiane Girard (1994) fueron los primeros en abordar los diferentes aspectos del servicio doméstico: su historia, las relaciones de trabajo en la cotidianidad, las perspectivas ideológicas, el debate con el feminismo, la organización sindical y las relaciones con el Estado. En esta perspectiva, las trabajadoras domésticas presentan mayores riesgos de ser vulnerables, pues al laborar / residir en un espacio privado y ajeno pueden presentarse humillaciones, acoso sexual y explotación laboral. En la mayoría de las investigaciones consultadas se hace hincapié en cómo el trabajo doméstico no reconoce los límites entre tiempo libre / tiempo de trabajo, ya que este tipo de labor, al no estar regulado legalmente en la mayoría de los países, permite la explotación a través de jornadas u horarios extendidos (Durin, 2012; Yeoh \& Huang, 2010).

Las relaciones de poder asimétricas que se dan en el empleo doméstico no solo tienen como eje el espacio privado del hogar, sino también el imaginario social 
construido alrededor de ese tipo de trabajo, que se configura sobre la desigualdad social y las diferencias de género, clase y fenotipo. En este sentido, esa actividad viene a ser ocupada en su mayoría por mujeres pobres y de escaso nivel educativo que sufren exclusión social (marginalización), exclusión económica (carencia de recursos) y exclusión social (precariedad laboral, vulneración de derechos). Tal segmentación del trabajo según clase o género ha implicado imaginarios y creencias estereotípicas hacia las trabajadoras domésticas como mujeres "sin educación aptas para la servidumbre", imaginarios que se han visto reforzados por la etnización del trabajo y la consideración del trabajo doméstico como una actividad que no requiere preparación ni cualificación alguna (Echeverría, 2015; Sánchez, 2001).

En cuanto a la relación entre empleo doméstico y género, las perspectivas feministas consideran que la revolución ocurrida en el mundo público no logró transformar el mundo privado, pues aunque la mujer cuenta con mayor acceso al mercado laboral, los hombres siguen sin intervenir en el espacio doméstico. Ante ello, las mujeres desempeñan un doble papel: son responsables de las tareas reproductivas y, a la vez, de las labores productivas fuera del hogar. Ello implica una doble y hasta triple jornada laboral, pues deben coordinar los quehaceres del ámbito privado y las responsabilidades del espacio público. Además, el servicio doméstico, al ser considerado como una labor exclusivamente femenina, reproduce las desigualdades entre géneros y construye una imagen de lo femenino limitada al ámbito doméstico (Hochschild \& Machung, 1989).

Ahora bien, ¿cómo abordar, entonces, la relación entre empleo doméstico y ciudad? En general, los pocos trabajos que han centrado su mirada en esta relación lo han hecho a partir del concepto de segregación espacial, acceso diferencial a la ciudad y movilidad cotidiana. Ejemplo de ello es el estudio llevado a cabo por Jan Marc Rottenbacher (2015) sobre la exclusión espacial que viven las empleadas domésticas en las playas de Lima, espacio en el cual coexisten personas de distintas clases sociales. Al respecto, el autor seńala que la ciudad está diseñada para la coexistencia intergrupal, mas no para el contacto, pues en lo espacial también se proyectan estereotipos, prejuicios y acciones hostiles contra sectores específicos de la población.

Por su parte, los análisis de Ana Belén Sánchez (2001) exponen cómo la segregación espacial de las empleadas domésticas sucede tanto en el espacio doméstico como en el espacio público. En este sentido, las mujeres se enfrentan con la restricción de espacios en su ámbito laboral (comedor, sala, habitaciones principales) y con la posibilidad restringida de acceder a ciertos lugares de la ciudad únicamente a través de su empleadora (centros comerciales, teatros, cines, etcétera), ya que al desconocer la trama urbana y su funcionamiento no pueden apropiarse de los espacios dispuestos en las metrópolis. Finalmente, el estudio de Carme Miralles-Guasch (1998) expone cómo las trabajadoras informales tienen mayores obstáculos de movilidad, pues sus destinos no se encuentran ubicados en áreas centrales y deben movilizarse mucho más que otras mujeres, situación que no implica conocimiento, apropiación o goce alguno del entorno urbano.

Así pues, estas investigaciones nos permiten comprender que la representación, apropiación y usos de la ciudad están condicionados por las dinámicas laborales y el conocimiento que los individuos tengan del espacio, siendo las empleadas 
domésticas uno de los sectores más vulnerables, dada la informalidad y feminización del sector, las largas jornadas laborales y las múltiples trayectorias espaciales. La marginalización, la segregación cognitiva y las diferencias en la experiencia espacial según posición socioeconómica, edad y género se conectan e inciden en las prácticas espaciales diarias. Ante ello, la movilidad se trasforma en un capital social que distingue y divide a la población urbana entre quienes pueden moverse con libertad, cómo, cuándo y a dónde quieran, y aquellas personas que no tienen muchas opciones -como las empleadas domésticas-, atrapadas como están en la "geometría de poder" (Massey, 1993).

\section{Atravesando la ciudad: desde los barrios marginales a las casas de "los ricos"}

\section{Marginalidad socioeconómica y espacial}

La labor doméstica ha estado relacionada con la migración. En la actualidad hay múltiples estudios sobre la migración internacional del trabajo en relación con la "globalización”, por ejemplo, el caso de mujeres colombianas trabajando como empleadas domésticas en diversos destinos internacionales (Bonilla Vélez \& Rodríguez López, 2013; Brevis, 2014; Parreńas, 2001; Salzinger, 1991). No obstante, y como señala Harzig (2006), han sido las desigualdades económicas, la migración rural-urbana y los cambios en la división internacional del trabajo los factores que históricamente han contribuido a la ampliación del sector doméstico. ${ }^{3}$

En Latinoamérica, este sector tiene unas características particulares que lo distinguen de los de otras latitudes: la migración a áreas urbanas en la región es desproporcionadamente femenina, lo que ha implicado un aumento de la mano de obra en las ciudades y una afectación a las estructuras familiares más generales (Rollins, 1985). Además, según García López (2012), diversos factores históricos han incidido en el aumento del empleo doméstico en el país; entre ellos, el crecimiento de las familias desde los años treinta del siglo xx y la presión económica resultante, que impulsó a los jóvenes a buscar trabajo a temprana edad para ayudar a la economía del hogar, lo cual a su vez resultó en un aumento de la migración a la ciudad. Además, hasta la mitad del siglo xx, pocas mujeres habían logrado trabajar en la esfera pública y solo algunas como meseras, vendedoras y prostitutas, razón por la cual la labor de empleada doméstica interna era un trabajo considerado apropiado para mujeres jóvenes. Según García López (2012), hay una estrecha vinculación entre el fenómeno del servicio doméstico, el éxodo rural y el crecimiento de las ciudades colombianas. El trabajo en casas de familia aumentó su demanda y oferta paralelamente al proceso de urbanización y modernización de Colombia. Hoy, según el Informe final Trabajadoras del hogar en Colombia (Centro de Cultura Popular José Antonio Galán, 2013), este sector laboral se ha precarizado -entre otros factores- por las altas tasas de desplazamiento rural a zonas urbanas y por el aumento de "mano de obra no calificada” en las ciudades. 
Al respecto, hemos encontrado a lo largo de nuestra investigación que la mayoría de nuestras interlocutoras proceden de zonas rurales: Cucutilla, Norte de Santander; Cayetano, Cundinamarca; Guateque, Boyacá; Aguadas, Caldas, entre otros. Estas mujeres migraron hacia Bogotá cuando eran niñas, con o sin sus familias, en búsqueda de trabajo y oportunidades. Oriundas de familias numerosas, las adolescentes tenían parientes (hermanas o hermanos) viviendo en la ciudad, quienes las recomendaron con vecinos y las acogieron en sus hogares. La mayoría de las mujeres iniciaron su vida laboral a una edad temprana, usualmente entre los diez y doce años. A esta edad, el empleo doméstico en modalidad de internas se presentó como el trabajo de más fácil acceso debido a la escasa formación educativa y la precariedad económica, ya que en su mayoría no habían finalizado la primaria, bien sea por las dificultades económicas o por el poco interés en continuar su educación. Tal ausencia de formación en otros campos es una de las principales razones por las cuales estas mujeres permanecieron en el sector doméstico ya adultas, aun cuando dicha labor les ofrece escasas oportunidades para salir de la precariedad económica (Centro de Cultura Popular José Antonio Galán, 2013). ${ }^{4}$

A lo anterior se suma la difícil situación económica, que ha arraigado a estas mujeres en barrios periféricos de la capital donde viven, como Ciudad Bolívar, Soacha, Suba o Tintal. De aquí tienen que atravesar cada día la ciudad para llegar a sus respectivos trabajos, ubicados usualmente al norte y occidente de la capital. Según Torres Tovar y Robles Joya (2014), en 2009 más que el 50\% de las viviendas de Bogotá estaban clasificadas como estrato 1 y 2 y localizadas mayoritariamente en la periferia de la ciudad, lejos de los principales centros de trabajo y de los bienes y servicios de la ciudad. Por esta razón, el sistema de transporte público asume un rol tan importante en la vida de las mujeres. En general se sienten cómodas en sus barrios, aunque frecuentemente asocian esta percepción a la costumbre y al conocimiento que tienen del espacio. Sin embargo, debido al alto índice de robos, microtráfico y delincuencia juvenil, en ocasiones se sienten vulnerables. ${ }^{5}$

A continuación expondremos en detalle la vida de dos de nuestras interlocutoras: Mireya y Floralba. ${ }^{6}$ Hemos seleccionado estos casos por representar situaciones típicas y recurrentes en la experiencia urbana de las empleadas domésticas. Además, al desarrollarse en distintas zonas de la ciudad dan cuenta de la heterogeneidad en las prácticas espaciales de nuestras interlocutoras y de las múltiples percepciones

4 El nivel educativo para conseguir empleo es más importante para mujeres que para hombres, como muestran las cifras más recientes de desempleo en Colombia. Según el Boletín Técnico del Departamento Administrativo Nacional de Estadística (DANE) (marzo 2017), la tasa de desempleo para mujeres con educación básica primaria es $9,8 \%$; con educación básica secundaria, 13,5\%, en comparación a hombres, con $5,8 \%$ y $8,6 \%$, respectivamente. En contraste, la tasa de desempleo para las mujeres que completaron la educación media fue $14,8 \%$, mientras para hombre era de $8,9 \%$. Y la tasa de desempleo de las mujeres que completaron la educación universitaria fue de $10,5 \%$ y la de los hombres, de $9,2 \%$.

5 Según el "Informe Calidad de Vida de Bogotá" (2017), las localidades con mayores niveles de microtráfico, atraco, extorsión y violencia sexual son Ciudad Bolívar, Bosa, Kennedy, Los Mártires y Santa Fe. En cuanto a tasas de homicidio, las localidades de Santafé $(64,2)$, Los Mártires $(54,2)$ y Ciudad Bolívar (35) tienen los índices más altos.

6 Pseudónimos empleados para proteger la privacidad de nuestras interlocutoras. 
que ellas tienen de los espacios geográficos. En este sentido, los casos seleccionados funcionan como cajas de resonancia a través de las cuales es posible visibilizar -en términos colectivos- las dinámicas subjetivas y espaciales del trabajo doméstico.

\section{Mireya}

Mireya es de Pitalito, Huila, pero debido a la difícil situación económica en la región migró hacia Bogotá junto a sus hermanos. Tiene 38 ańos, tres hijos (de 16, 9 y 7 ańos, respectivamente) y trabaja hace quince ańos como empleada doméstica. Ingresó al sector gracias a recomendaciones de familiares y amigos y ha permanecido desde entonces en este tipo de empleo, que para ella es garantía de tranquilidad al ofrecerle la disponibilidad de tiempo necesaria para "hacerse cargo de su familia". Actualmente trabaja de lunes a sábado en dos casas distintas: una ubicada en el barrio La Castellana (noroccidente) y otra en el barrio Colina (norte).

Mireya vive en el barrio Berlín, parte de la UPZ (Unidades de Planeamiento Zonal) Tibabuyes de Suba, entre los límites de Bogotá y los de la Sabana. Fundado en 1991 como proyecto de urbanización de la Inmobiliaria La Nacional en zona suburbana, los predios del barrio solo fueron legalizados formalmente en 1998 . Hoy en día las casas son de ladrillo y de varios pisos, pero su origen "pirata" todavía es evidente: en su mayoría las calles no están pavimentadas y suelen inundarse con los aguaceros. Además, no todos los sectores del barrio tienen acueducto y alcantarillado, por lo cual esta zona es definida por la planeación urbana como estrato 2 .

En general, la localidad está marcada por la inseguridad, pues, según la líder comunitaria del sector, los residentes de Berlín que salen temprano a trabajar-como las empleadas domésticas- son afectados de manera recurrente por robos y atracos:

Nos preocupa que la población del barrio, gente humilde y trabajadora que sale
cada día a buscar el sustento de su familia desde las cuatro de la mañana, sea la más
afectada por los robos y atracos, especialmente a esa hora cuando aún no amanece.
Le pedimos al alcalde mayor, al alcalde local y a la policía que intensifiquen su
presencia en nuestro barrio. (Jiménez, s.f.)

Sin embargo, las estrategias de cuadrantes, alarmas comunitarias y frentes de seguridad se quedan cortas frente a la delincuencia organizada, ante lo cual muchos de los habitantes del lugar han decidido mudarse de barrio o tratar -en la medida de lo posible- de acceder a un trabajo cuya jornada laboral sea más flexible.

Aparte de la seguridad, los residentes de Berlín reclaman que falta mucho por intervenir en términos de vías. También comentan que los fines de semana el agua no sube hasta los segundos y terceros pisos de sus casas. De igual modo, esperan una intervención al río Bogotá, el cual inunda frecuentemente el barrio y afecta la salud de sus residentes (Jiménez, s.f.).

En este espacio se ubica la casa de Mireya, la cual tiene tres pisos y fue construida por su suegro tres décadas atrás. En el primer piso está el negocio de sus suegros (tienda-cafetería) y en el segundo, su residencia. Al subir por unas escaleras empinadas, oscuras y angostas, llegamos al tercer piso. Allí vive la familia de Mireya (esposo y tres hijos) y la familia de su cuńado (esposa y dos hijos). El apartamento de Mireya mide apenas 40 metros cuadrados e incluye sala, cocina, comedor y dos 
cuartos. El baño está ubicado en el corredor y es de uso compartido entre las dos familias. Los techos bajos de la construcción y la escasa luz de las habitaciones generan una sensación de ahogamiento. Sin embargo y pese a los problemas de hacinamiento, Mireya se siente cómoda y en compañía en su hogar, pues "es casa propia” y la mayoría de su familia vive en los alrededores.

En la cotidianidad de Mireya, uno de los desafíos más grandes es el servicio de transporte. La localidad no cuenta con suficientes rutas y con acceso a distintas ofertas de movilidad, ante lo cual Mireya suele demorarse entre una hora y hora y media para llegar desde su hogar hasta su trabajo en el norte de la capital (figura 2). Además, el tráfico en Suba suele ser pesado y lento, pues esta localidad solo cuenta con dos vías de acceso: la avenida Suba y la calle 80. Por otra parte, el sector es limítrofe con varios municipios de Cundinamarca (Cota, Chía, Cogua, etcétera) y recibe gran cantidad de transporte intermunicipal, situación que complica el tránsito y la movilidad para los residentes de la zona.

FIgURA 2 Recorridos de Mireya

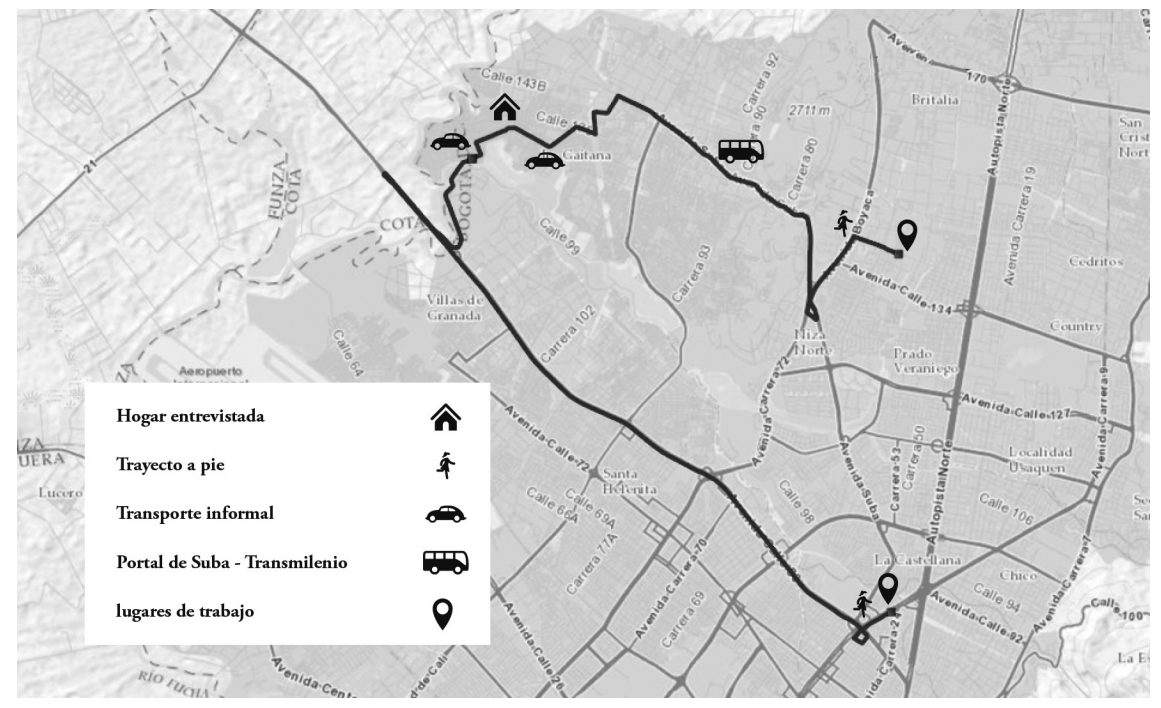

FUENTE ELABORACIÓN PROPIA

Frente a estas problemáticas de transporte y las continuas quejas de la ciudadanía relacionadas con su no contar con las mismas garantías de acceso y disfrute de la ciudad, la Alcaldía Mayor de Bogotá inauguró en 2006 un Portal de Transmilenio en la localidad de Suba. No obstante, y como muestra Beuf $(2011$, 2012), las líneas y estaciones de este sistema masivo están definidas frecuentemente por intereses económicos del desarrollo urbano. De esta manera no sorprende que, en los márgenes de esta localidad, donde queda Berlín, las opciones de transporte sigan siendo limitadas. Además, y según Mireya, en el contexto de la expansión de la red de Transmilenio quitaron varias rutas de los buses regulares, lo que dejó a los residentes con menos opciones para transitar por la ciudad: 
Los alcaldes creen que Transmilenio lo mueve todo, y es mentira. Mueve una parte, pero ¿el resto de gente qué? Por ejemplo, en estos barrios, que es donde sale más gente, no hay sitp, ${ }^{7}$ no hay alimentador y no hay buenas rutas de transporte. Además, los alcaldes, al centrarse únicamente en Transmilenio, no miran otras opciones de transporte o cómo mejorar el que hay. (Comunicación personal, Mireya, marzo 11, 2017)

Frente a ello, Mireya ha optado por recurrir a estrategias que le permitan un acceso más fácil y rápido a la ciudad: camina varios trayectos desde su hogar al trabajo y emplea, en la mayoría de los casos, los buses regulares para movilizarse, ya que las rutas que estos manejan la dejan más cerca de sus destinos. Además, dice sentirse más cómoda y segura en los buses que en el sistema de Transmilenio, pues ha sufrido en varias ocasiones acoso sexual y ha sido testigo a su vez de atracos dentro del sistema.

Sin embargo y pese a las estrategias desplegadas por Mireya, la ciudad sigue siendo un espacio desconocido para ella. Al no contar con oferta suficiente para movilizarse, no ha tenido la oportunidad de transitar y conocer la ciudad. En este sentido, los lugares de recreación, compras y ocio se circunscriben a la localidad donde reside.

\section{Floralba}

Floraba es oriunda de Guateque, Boyacá. Tiene 51 años y cinco hijos (de 31, 26, 25, 19 y 16 años). Migró hacia la ciudad cuando tenía ocho años, pues quedó huérfana a temprana edad y sin nadie que cuidara de ella. En tal situación y gracias a la recomendación de una vecina, empezó a trabajar a los nueve años como empleada doméstica y se desempeña desde entonces en ese sector. Actualmente trabaja de lunes a viernes en cuatro casas distintas, todas ellas ubicadas en el norte de la ciudad (localidad de Usaquén).

Para desplazarse hacia sus distintos lugares de trabajo, Floralba gasta en promedio cuatro horas diarias (ida y vuelta), ya que reside en Ciudad Bolívar, una de las localidades más distantes y periféricas de la ciudad (figura 3). La localidad es un asentamiento urbano no planeado que empezó a poblarse a partir de la década de los cincuenta del siglo pasado. Por ese entonces empezaron a surgir los primeros asentamientos en esta parte de la ciudad, siendo los primeros barrios fundados por personas que huían del conflicto armado y que migraron hacia Bogotá por falta de oportunidades económicas en sus lugares de origen.

Además, este sector se caracteriza por tener altos niveles de pobreza y el índice de condiciones de vida más bajo de la localidad. Al respecto, los resultados de la Encuesta Multipropósito realizada en 2014 por el Departamento Administrativo Nacional de Estadística (DANE, 2014) exponen que en el 68,4\% de los hogares de Ciudad Bolívar los ingresos solo alcanzan para cubrir los gastos mínimos. Además, y tal como nos comentó Floralba, en su mayoría los barrios del sector no cuentan con acceso a servicios públicos ni a vías de transporte. En su barrio (Altos de Cazucá),

7 El Sistema Integrado de Transporte Público (sITP) articula el Transmilenio con rutas de buses regulares y alimentadores, con lo que se intenta lograr una mayor cobertura y reducir el tiempo de movilidad en la ciudad. 
el agua potable fue instalada hacia mediados de 2015: "hace dos ańos que tenemos agua, antes nos llegaba por manguera cada mes o cada dos meses. Sufríamos mucho por eso, pero ya tenemos agua potable, gracias a Dios" (comunicación personal, Florabla, abril 10, 2017). Este acceso a los servicios públicos ha incidido en el poblamiento del sector, ya que desde entonces el barrio se ha densificado y las condiciones de seguridad han mejorado notablemente. No obstante, la localidad sigue siendo, según el Informe de Calidad de Vida (2017), uno de los sectores con las mayores tasas de homicidios y delitos contra la convivencia de la ciudad.

\section{Figura 3 Recorridos de Floralba}

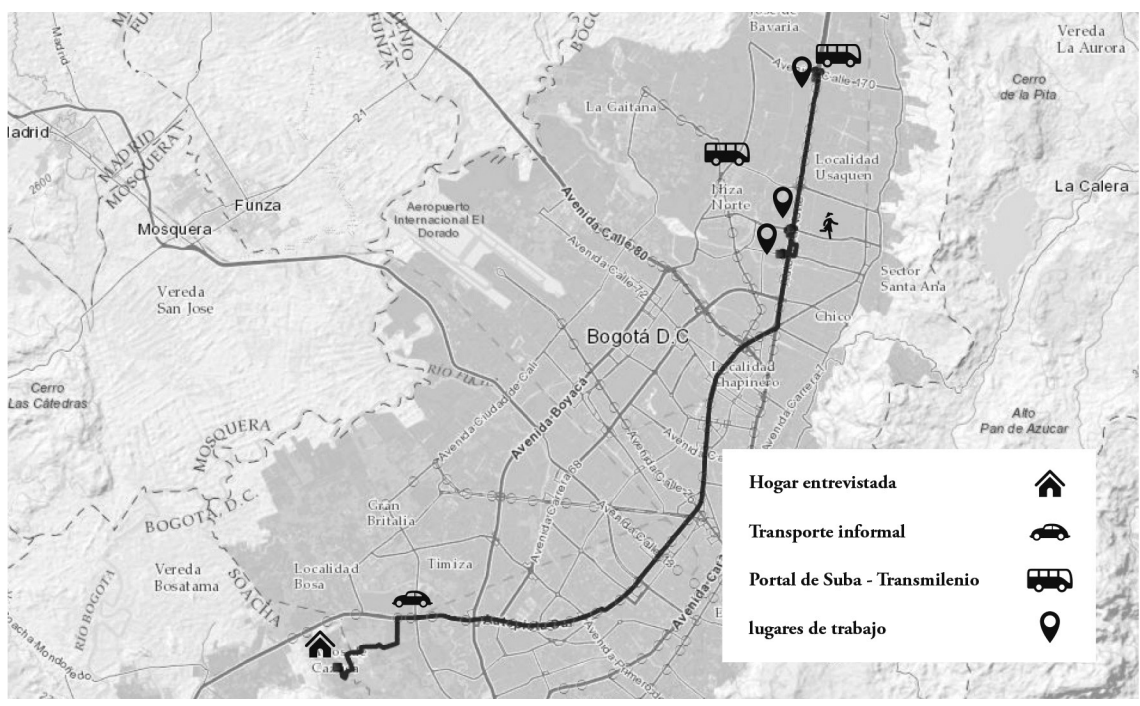

FUENTE ELABORACIÓN PROPIA

Pese a lo dicho, Floralba reside en la localidad desde hace dieciséis años, pues allí logró construir su casa propia tras ańos de esfuerzos y sacrificios. Sin embargo, suele sentirse insegura en esta parte de la ciudad ya que ha sido víctima de robos y atracos en reiteradas ocasiones. $\mathrm{Al}$ respecto, comenta lo siguiente:

La zona es muy insegura, hay mucho ladrón. Por ejemplo, uno va por la calle y -como dice el cuento- los ojos en la espalda y una mano adelante y otra atrás, porque en cualquier momento le cogen a uno la cartera y la amenazan con cuchillo, revólver o con lo que sea. (Comunicación personal, Floralba, abril 10, 2017)

Esta situación de inseguridad impide el tránsito por el sector y la apropiación de los espacios públicos allí construidos. En tales circunstancias, los residentes del barrio han recurrido a varias estrategias de protección, como la creación de redes de apoyo entre vecinos y familiares. Ejemplo de ello es Floralba: para llegar a su trabajo ubicado en la localidad de Usaquén debe tomar el bus a las 4:30 a.m. Para evitar robos sale hasta el paradero acompańada de vecinos o familiares, los que se organizan cada semana en grupos de acompańamiento. 
Sumado a estas dificultades en materia de seguridad, el acceso a transporte en el barrio es restringido, pues las carreteras no se encuentran pavimentadas y el Transmilenio no tiene cobertura en esta parte de la ciudad. Floralba suele tomar dos buses por trayecto: de su casa toma una buseta regular ${ }^{8}$ hasta la autopista sur, de allí camina hasta el Portal del Sur y toma el Transmilenio hacia el norte. Demora en promedio dos horas por trayecto, pues la congestión del sistema, los constantes pleitos entre los pasajeros y el poco flujo de vehículos -su insuficiente cantidad en relación con la necesidad y demanda- impide una movilidad ágil y eficaz a través de la ciudad. Además, el transporte suele ser costoso en relación con los ingresos de Floralba, pues en promedio gasta nueve mil pesos diarios (aproximadamente US\$3).

La apropiación y uso del espacio es, así, reducida, pues tal y como plantea la geografía marxista, la movilidad en la ciudad depende en gran medida de factores socioeconómicos. Además, la localidad, al ubicarse en las periferias y contar con pocos espacios de esparcimiento y recreación, queda al margen de las otras dinámicas urbanas que se desarrollan. Floralba experimenta esta segregación en la imposibilidad de acceder a otros espacios de la ciudad debido a las precarias ofertas de transporte. Además, movilizarse es gastar dinero, ante lo cual varias de las mujeres prefieren circunscribir sus actividades a sectores cercanos. En este sentido y desde la perspectiva de las mujeres, la ciudad no está hecha para su disfrute y apropiación, sino únicamente para el desarrollo de labores productivas.

\section{Atravesando: la (in)movilidad}

Bogotá se caracteriza por su patrón de segregación particular: en el sur y las periferias se ubican los barrios obreros, mientras en el norte de la ciudad están localizadas zonas con un alto nivel de vida. Sectores monofuncionales (zonas administrativas, industriales, residenciales y de esparcimiento) implican una inadecuación entre los lugares de residencia y la localización de los empleos. El efecto es la segregación habitual, efecto de la dificultad para movilizarse, agravada por los largos desplazamientos diarios que exigen las tareas de la vida cotidiana (Dureau, 2013).

En promedio, las mujeres entrevistadas gastan entre una hora y media y dos horas por trayecto (casa-trabajo, trabajo-casa), es decir, de tres a cuatro horas diarias en el transporte público. Según estas mujeres, el transporte en la ciudad no cubre las necesidades de los usuarios, pues no hay flujo constante de vehículos ni rutas suficientes. En este sentido, pese a que el Sistema de Transporte Masivo Transmilenio surgió a finales del 2000 como una alternativa para superar la congestión vial y la segregación espacial de ciertos sectores de la capital, su implementación y funcionamiento han supuesto -en algunos casos- mayores dificultades y obstáculos en la movilidad e integración espacial de la ciudad. El Transmilenio cuenta con 138 estaciones y nueve portales, ${ }^{9}$ distribuidos a través de las principales avenidas de la ciudad.

El sistema de transporte público regular es un servicio que ofrecen operadores privados no integrados al sistema de Transmilenio. Este servicio carece de paraderos fijos, tiene mayor cobertura y la tarifa de servicio es menor, pues no requiere de infraestructura especializada para su funcionamiento.

9 Portal El Dorado, Portal Las Américas, Portal Norte, Portal 20 de Julio, Portal 80, Portal Suba, Portal Sur, Portal Tunal y Portal Usme. 
Este sistema cuenta a su vez con servicios urbanos, complementarios y especiales, los cuales buscan integrar la ciudad mediante una red de servicios más amplia. Si bien el sistema se ha extendido mediante la oferta de servicios complementarios, como el Sistema Integrado de Transporte Público (SITP) y los buses alimentadores, las rutas que estos manejan no tienen la cobertura necesaria para incluir los sectores marginales y periféricos de la capital. Ante ello, los buses regulares son una opción viable para personas que viven lejos de las líneas de Transmilenio o carecen de la capacidad económica necesaria para acceder a este sistema. Además, pese a que el Transmilenio cuenta con carriles exclusivos, el tiempo de espera para realizar transferencias suele ser de 30 a 45 minutos, ante lo cual las mujeres entrevistadas califican el sistema como ineficiente y costoso. Sumado a ello, la inseguridad, el acoso sexual y la congestión al interior del sistema las llevan a preferir medios alternativos, como buses o colectivos, pues estos tienen mayor cubrimiento espacial y, a la vez, según las usuarias, garantizan mayor seguridad y comodidad.

No obstante, la oferta de transporte no es el único factor que incide en la movilidad de las mujeres incluidas en este estudio. A partir del análisis de las trayectorias individuales de movilidad, logramos definir algunas pautas de movimiento e identificar el nivel de inserción territorial de varios sectores urbanos. En términos generales, la mayoría de las mujeres desconoce la ciudad, ya que sus actividades se circunscriben al barrio donde residen y al sector donde trabajan, y ello pese a ser uno de los sectores laborales con mayor potencial de movimiento. En estas circunstancias, podemos afirmar que las empleadas domésticas tienen "modos de habitar menguados", pues sus patrones de movilidad y el diseño urbano impiden el uso, acceso y disfrute de la ciudad (Lindón, 2006). El espacio, más que un entorno físico, es un escenario en el cual se representan los roles asignados socialmente, así como el poder simbólico de diferentes grupos sociales, pues la presencia física no implica la visibilidad social de un colectivo ni la posibilidad real de desplazarse en la ciudad (Sánchez, 2001).

Las dificultades en la movilidad que se han señalado no solo afectan la calidad de vida de las empleadas domésticas, sino también las dinámicas en su núcleo familiar, pues el tiempo con sus hijos y compańeros se reduce drásticamente por las dificultades para desplazarse rápida y efectivamente a través de la ciudad.

\section{Trabajar: estar en "el norte"}

Pese a que la mayoría de las mujeres labora en el norte de la ciudad, casi ninguna de ellas conoce este espacio con propiedad más allá del hogar donde trabajan. No reconocen en este lugar un "espacio para ellas", pues se sienten externas a él. Tal percepción es producto de los imaginarios urbanos construidos alrededor de ciertas zonas y la estratificación de la ciudad, pues las mujeres se segregan espacialmente en estos lugares, ya que sienten que "no pertenecen allí" por su origen urbano, su proveniencia de barrios periféricos y marginales.

La discriminación desempeña un papel fundamental en la percepción de los espacios. Si bien la mayoría de las mujeres no ha sufrido exclusión, algunas admiten haber sido objeto de burlas o señalamientos al transitar con el uniforme puesto por los barrios donde trabajan. Otras también han sufrido actos de discriminación 
en negocios y centros comerciales. Ejemplo de ello es el caso de Lucía, empleada doméstica en el sector de Chapinero. Un día fue a almorzar con su hija cerca de su trabajo, y en el restaurante le preguntaron más de tres veces si tenía dinero suficiente para pagar la cuenta. Lucía relata que en varias ocasiones ha sentido que la gente de estas zonas la observa con desdén, pues consideran que ella no pertenece al lugar ni puede acceder a los mismos servicios que ellos. Pese a esto, las mujeres se sienten orgullosas de su labor y consideran que estos casos son esporádicos y no representan las dinámicas de la ciudad en sí.

Asímismo, disfrutan de esta parte de la ciudad, pues allí se sienten más cómodas y seguras. Además, resaltan la belleza del lugar, la amplia vigilancia en la zona y la multiplicidad de espacios verdes y de recreación que allí encuentran. Esta percepción da cuenta de la distribución inequitativa de bienes materiales e inmateriales en la ciudad y la injusticia territorial que se construye a partir de la planeación urbana (Harvey, 1977), pues contrario a las zonas 'estrato 6', los barrios periféricos no cuentan con un sistema de seguridad continuo, áreas verdes y zonas de esparcimiento. Así pues, el norte de la ciudad es percibido como un espacio 'ideal' al cual, sin embargo, no se tiene acceso.

\section{Conclusiones: la triple otredad de las empleadas domésticas}

Como ilustran las viñetas presentadas, los procesos de movilidad son heterogéneos, pues dependen en gran medida de factores socioeconómicos, de género y ocupación. Las empleadas domésticas en la ciudad de Bogotá no solo deben enfrentarse a la informalidad y discriminación que sufre el sector, sino al reto de la movilidad, pues la mayoría vive en barrios marginales de Bogotá, marginales tanto en términos de ubicación como de la concepción popular.

Estos retos de movilidad tienen estrecha relación con la estratificación inscrita en las políticas del espacio: las localidades urbanas están clasificadas según su estatus socioeconómico. De hecho, el sistema de estratos se ha vuelto parte de la cultura urbana: sirve como descripción de la apariencia, de la calidad y de los servicios de ciertas áreas de la ciudad. La ciudad es transitada y percibida a través de estos imaginarios, lo que impide a varios sectores de la población acceder con igualdad de posibilidades a los bienes y servicios que ella ofrece. Esto permite señalar que la ciudad se ha planeado con base en el deseo de excluir y privatizar, no de integrar. Esta producción de espacios disociados y la ciudad dividida en sectores monofuncionales han generado la inadecuación, según Dureau (2013), entre la localización de los empleos, de los servicios y de los lugares de residencia; es decir, un desencuentro espacial (spatial mismatch) que obliga a un mayor número de desplazamientos.

Ahora bien, en la vida de las empleadas esta estratificación socioespacial y conceptual tiene un efecto muy concreto: ellas son los "otros" en tres sentidos: por su residencia, por su origen de clase y por el viaje en sí mismo: de barrios marginales a barrios acaudalados. Tales características se agudizan por el origen rural de muchas de ellas. Una dimensión de otredad que se agrega a las anteriores es la apariencia física de las mujeres, es decir, la corporeidad en el espacio. Esta es parte de la distinción de clase, que en el contexto colombiano corresponde a la asociación 
explícita entre espacio y poder socioeconómico. Y aunque muchas de las empleadas consideran apropiado vestir uniforme para trabajar (para no gastar su ropa propia), ello las marca aún más en el contexto socioespacial de los lugares de trabajo, donde en ocasiones son excluidas y discriminadas.

Es así que las travesías de las mujeres del campo a la ciudad, su residencia en los barrios marginales y el tránsito desde sus hogares hacia los sectores más lujosos de la urbe, implican para las mujeres estar marcadas -excluidas- de tres maneras: estrato / clase, género, y ubicación en el paisaje urbano. Estas dimensiones confluyen para formar la experiencia urbana de las empleadas domésticas e impactar en ella. Volviendo al concepto de motility, las posibilidades de movilidad para las empleadas domésticas son expresión clara de la triple exclusión señalada: no tienen opción ni voluntad sobre sus trayectorias (movilidad obligada) y, en términos de formas de transporte, no pueden decidir sobre su tiempo (cuándo viajar y qué tiempo gastar). Poco sorprende, entonces, que la mayoría de las mujeres opten por no viajar en su (poco) tiempo libre. Mientras los estratos más altos tienen opciones de movilidad, las mujeres de los estratos bajos están arraigadas a un sistema de transporte y una lógica urbana pensada para las clases medias y altas. Sus posibilidades se limitan a decidir qué bus o ruta exacta toman. Al mismo tiempo, es con estas decisiones que las mujeres también producen el espacio, en el sentido signado por Lefebvre: escogen ciertas rutas sobre otras, un medio de transporte en lugar de otro, o deciden caminar en vez de trasbordar o esperar. Además, sus experiencias subjetivas y sus discursos sobre la ciudad y el sistema de transporte también contribuyen a esta producción del espacio de la capital.

La movilidad se muestra, así, como un capital que distingue y marca las diferentes clases urbanas. La motility de las empleadas domésticas es una expresión de la estratificación socioeconómica y espacial de la ciudad, ya que las formas en que se configura la otredad afecta fuertemente su capital de movilidad. Falta de motility implica falta de acceso a recursos, lugares o personas, o un mayor costo para acceder a ellos; y significa, por ende, exclusión social. Es aquí donde se evidencia con mayor fuerza la importancia de la planeación urbana, ya que esta podría aliviar los desafíos diarios de las empleadas domésticas y otros trabajadores del sector informal. Si bien es difícil superar la estratificación socioeconómica y espacial en la ciudad, un sistema de transporte bien planeado podría equilibrar las desigualdades urbanas.

\section{Agradecimientos}

Esta investigación fue financiada por la Universidad de los Andes, Bogotá, 2015-2018. Agradecemos a María José Álvarez-Rivadulla, Diana Ojeda y Sergio Montero por sus comentarios en relación con la investigación. 


\section{Referencias bibliográficas}

Alcaldía Local de Ciudad Bolívar, Alcaldía Mayor de Bogotá (s.f.). Conociendo mi localidad. [Página web]. https://bit.ly/2wKKqBY

Ares, S. (2010). Espacio de vida y movilidad territorial habitual en Chapadmalal, Buenos Aires, Argentina. Revista Colombiana de Geografía, 19, 27-40. https://doi.org/10.15446/ rcdg.n19.16840

Bailly, A. (1989). Lo imaginario espacial y la geografía: en defensa de la geografía de representaciones. Anales de Geografia, 9, 11-19. https://bit.ly/2CATGe6

Beuf, A. (2011). Nuevas centralidades y acceso a la ciudad en las periferias bogotanas. Bulletin de l'Institut français d'études andines, 40(1), 147-178. http://journals.openedition.org/ bifea/ 1663

Beuf, A. (2012). De las luchas urbanas a las grandes inversiones. La nueva urbanidad periférica en Bogotá. Bulletin de l'Institut français d'études andines, 41(3), 473-501. http:// journals.openedition.org/bifea/344

Bonilla Vélez, G. \& Rodríguez López, M. (2013). Migración femenina desde el Caribe colombiano. Una mirada a sus espacios laborales en destino. Memorias, Revista digital de Historia y Arqueología desde el Caribe colombiano, 21, 152-178. http://www.redalyc. org/articulo.oa?id=85529051011

Brevis, H. (2014). Empleo doméstico en Navarra. Universum, 29(1), 139-152. http://doi. org/10.4067/S0718-23762014000100008

Britto da Motta, A. (1985). Emprego domestico em Salvador - Relatorio final da pesquisa. Salvador: Universidad Federal de Bahía.

Centro de Cultura Popular José Antonio Galán (2013). Informe Final Trabajadoras del Hogar en Colombia, https://bit.ly/2C3zsZo

Chaney, E. \& García, M. (coords.). (1989). Muchachas no more, household workers in Latin America and the Caribbean. Filadelfia, PA: Temple University Press. https://bit. ly/2OSrgSN

Delgado, M. (2007). Sociedades movedizas. Barcelona: Anagrama. https://bit.ly/2IO1nxW

Departamento Administrativo Nacional de Estadística (DANE) (2014). Encuesta multipropósito. https://bit.ly/2uI0ala

Departamento Administrativo Nacional de Estadística (DANE) (2017, marzo 24). Boletín técnico. https://bit.ly/2PkSjms

Dureau, F. (2013). Cambios urbanos, transporte masivo y desigualdades socio-territoriales en unos barrios del occidente de Bogotá. Cuadernos de Vivienda y Urbanismo, 6(11), 44-67. https://bit.ly/2RzM9QY

Durin, S. (2012). Servicio doméstico de planta y discriminación en el área metropolitana de Monterrey. Relaciones, 93-129. https://bit.ly/2RCaYvv

Echeverría, R. (2015). Mujeres indígenas rurales trabajadoras domésticas: exclusión social en el espacio urbano de Mérida, Yucatán. Nóesis, Revista de Ciencias Sociales y Humanidades, Volumen especial, 93-110. https://bit.ly/2y8JYvM

Espinoza, J. (2013). Espacios objetivos y subjetivos de la movilidad cotidiana urbana. Revista Cartográfica, 89, 103-118. https://bit.ly/2Nxwuyq 
Fuentes Durán, H. (2010). Desigualdad, segregación socio-espacial y precios del suelo en la Ciudad Latinoamericana. El caso de Bogotá en los años noventa. UIS Humanidades, 38(1), 109-126. https://bit.ly/2NvDM5U

García López, A. C. (2012). Trabajo a cambio de pertenencia, empleadas domésticas en Bogotá, 1950-1980. Revista Grafia, 9, 159-174. https://bit.ly/2OJLXzZ

Girard, C. (1994). Cidadania e cultura o universo das empregadas domesticas em Brasilia (19791990). Tesis de doctorado. Brasilia: Universidade de Brasilia. https://bit.ly/2Obxttb

Hanson, S. (2010). Gender and mobility: New approaches for informing sustainability. Gender, Place \& Culture: A Journal of Feminist Geography, 17(1), 5-23. https://doi. org/10.1080/09663690903498225

Harvey, D. (1977). Urbanismo y desigualdad social. Madrid: Siglo XxI Editores. https://bit. ly/2PhIvJY

Harzig, C. (2006). Domestics of the world (unite?): Labor migration systems and personal trajectories of household workers in historical and global perspective. Journal of American Ethnic History, 25(2/3), 48-73. https://www.jstor.org/stable/27501688

Hiernaux, D. (2007). Los imaginarios urbanos: de la teoría y los aterrizajes en los estudios urbanos. EURE, 33(99), 17-30. http://doi.org/10.4067/S0250-71612007000200003

Hochschild, A. \& Machung, A. (1989). The Second Shift: working parents and the revolution at home. Nueva York: Viking.

Informe Calidad de Vida de Bogotá (2017). Bogotá Cómo Vamos. https://bit.ly/2RywCRn

Järv, O., (2014). Ethnic differences in activity spaces as a characteristic of segregation. Urban Studies, 52(14), 2680-2698. https://doi.org/10.1177/0042098014550459

Jelin, E. (1977). Migrants and women who wait. Migration and labor force participation of Latin American woman: The domestic servants in the cities. Signs, 3(1), 129-141. https://bit.ly/2yoX7A8

Jensen, K. (2014). Space-time geography of female live-in child domestic workers in Dhaka, Bangladesh. Children's Geographies, 12(2), 154-169. https://bit.ly/2E97A8R

Jiménez, G. R. (s.f.). Los madrugadores, los mayores afectados por la inseguridad en el barrio Berlín de Suba. Suba Alternativa. https://bit.ly/2pHQOn6

Jirón, P. (2007). Implicancias de género en las experiencias de movilidad cotidiana urbana en Santiago de Chile. Revista Venezolana de Estudios de la Mujer, 12(28), 173-197. https:// bit.ly/2QCuuXr

Kaufmann, V., Bergman, M. \& Joye, D. (2004). Motility: Mobility as capital. International Journal of Urban and Regional Research, 28(4), 745-756. https://doi.org/10.1111/ j.0309-1317.2004.00549.x

Kaztman, R. (2001). Seducidos y abandonados: el aislamiento social de los pobres urbanos. Revista de la CEPAL, 75, 171-189. https://bit.ly/2ObpsEF

Kofes, S. (1990). Mulher, mulheres: diferencia e identidade nas armadilhas da igualdade $e$ desigualdade: interacao e relacao entre patroas e empregadas domesticas. Tesis de doctorado. San Pablo: Universidad de San Pablo.

Lefebvre, H. (1991). The production of space. Oxford: Blackwell.

León, M. (1982). Acciones para transformar las condiciones socio-laborales del servicio doméstico en Colombia. Bogotá: Fundación Ford. http://doi.org/10.7440/res45.2013.16

Lindón, A. (2000). La vida cotidiana y su espacio-temporalidad. Barcelona: Anthropos. 
Lindón, A. (2006). La espacialidad de la vida cotidiana: Hologramas socio-territoriales de la cotidianeidad urbana. En J. Nogué i Font \& J. Romero (coords.), Las otras geografias (pp. 425-446). Valencia: Tirant Lo Blanch.

"Los estratos 'acentúan la segregación y los estereotipos': unAL". (2016, enero 14). Dinero. https://bit.ly/2OdBA8k

Massey, D. (1993). Power-geometry and a progressive sense of place. En J. Bird, B. Curtis, T. Putnam, G. Robertson \& L. Tickner (coords.), Mapping the futures: Local cultures, global change (pp. 59-69). Londres: Routledge.

Miralles-Guasch, C. (1998). La movilidad de las mujeres en la ciudad: un análisis desde la ecología urbana. Ecología Politica, 15, 123-130. https://bit.ly/2RBrEDp

Molina, I. (2006). Romper barreras, género y espacio en el campo y la ciudad. Santiago: Ediciones El Tercer Actor. https://bit.ly/2E3EYhc

Parreñas, R. (2001). Servants of globalization: Women, migration, and domestic work. Stanford, CA: Stanford University Press.

Rico de Alonso, A. (2001). Familia, género, pobreza urbana en Colombia: supervivencia y futuro. Papel Político, 13, 115-135.

Rincón Reina, M. \& Zanger, P. (2013). Barrio BERLIN de la Localidad de SUBA, Distrito Capital BOGOTÁ. CID Online Verlag. http://berlin-suba.blogspot.com.co

Roca, M. (2012). Segregación social: distribución y construcción espacial en Lima metropolitana. Espacio y Desarrollo, 24, 119-134. https://bit.ly/2OKlqm1

Rojas, G. \& Toledo, M. (2014). Reproducción social estratificada: el trabajo doméstico remunerado en México y la interacción entre mujeres de estratos medios y populares. En L. Gandini \& M. Padrón (coords.), Población y trabajo en América Latina. Abordajes teórico-metodológicos y tendencias empíricas recientes (pp. 403-441). México: Asociación Latinoamericana de Población (ALAP). https://bit.ly/2CwDri1

Rollins, J. (1985). Between women: Domestics and their employers. Filadelfia, PA: Temple University Press.

Rottenbacher, J. (2015). Trato discriminatorio hacia empleadas domésticas y segregación socioespacial en balnearios de Lima. Revista de Psicología, 33(2), 241-275. https://bit. ly/2ObKq6z

Rubbo, A. \& Taussig, M. (1983). Up off their knees: Servanthood in Southwest Colombia. Latin American Perspectives, 10(4) (Health, Violence, Race and Class), 5-23. https:// doi.org/10.1177/0094582X8301000402

Ruiz, J. (2016). La persistencia de la segregación y la desigualdad en barrios socialmente diversos: un estudio de caso en La Florida, Santiago. EURE, 42(125), 81-108. http:// doi.org/10.4067/S0250-71612016000100004

Salzinger, L. (1991). A maid by any other name: The transformation of 'dirty work' by Central American immigrants. En M. Buraway et al. (coords.), Ethnography unbound: Power and resistance in the modern metropolis (pp. 139-160). Berkeley, CA: University of California Press.

Sánchez, A. (2001). Los usos del espacio urbano y el proceso de integración de la mujer migrante. Revista Electrónica de Geografía y Ciencias Sociales, 94. http://www.ub.edu/ geocrit/sn-94-101.htm 
Santillán, A. (2015). Imaginarios urbanos y segregación socioespacial. Un estudio de caso sobre Quito. Cuadernos de Vivienda y Urbanismo, 8(16), 246-263. https://doi.org/10.11144/ Javeriana.cvu8-16.iuss

Saraví, G. (2008). Mundos aislados: segregación urbana y desigualdad en la ciudad de México. EURE, 34(103), 93-110. http://doi.org/10.4067/S0250-71612008000300005

Sheller, M. \& Urry, J. (2006). The new mobilities paradigm. Environment and Planning A, 38, 207-226. https://doi.org/10.1068/a37268

Sheller, M. \& Urry, J. (2016). Mobilizing the new mobilities paradigm. Applied Mobilities, 1(1), 10-25. https://doi.org/10.1080/23800127.2016.1151216

Silvey, R. (2006). Geographies of gender and migration: spatializing social difference. $I M R$, 40(1), 64-81. https://doi.org/10.1111/j.1747-7379.2006.00003.x

Sitp.gov.co. (s.f.). Información general. http://www.sitp.gov.co/Publicaciones/el_sistema/ informacion_general

Smith, N. (2000). Capitalism. The Dictionary of Human Geography. Oxford: Blackwell.

Soja, E. (2008). Postmetrópolis. Estudios criticos sobre las ciudades y las regiones. Madrid: Traficantes de Sueños.

Soto, P. (2013). Repensar las prácticas espaciales: rupturas y continuidades en la experiencia cotidiana de mujeres urbanas de la Ciudad de México. Revista Latinoamericana de Geografia e Genero, 4(2), 2-12. https://doi.org/10.5212/Rlagg.v.4.i2.002012

Soto, P. (2017). Diferencias de género en la movilidad urbana. Las experiencias de viaje de las mujeres en el Metro de la Ciudad de México. Revista Transporte y Territorio, 16, 127146. https://bit.ly/2IJSDZu

Téllez Oliveros, V. (2014, 22 de junio). La pobreza oculta de Bogotá. El Espectador. https://bit. $\mathrm{ly} / \mathrm{V} 49 \mathrm{PPJ}$

Thibert, J. \& Osorio, A. (2014). Urban segregation and Metropolitics in Latin America: The case of Bogotá, Colombia. International Journal of Urban and Regional Research, 38(4), 1319-1343. https://doi.org/10.1111/1468-2427.12021

Torres Tovar, C. \& Robles Joya, S. (2014). Estrategias de inclusión-exclusión de la ciudad colombiana autoproducida mediante políticas de reasentamiento barrial. Bulletin de l'Institut français d'études andines, 43(3). https://journals.openedition.org/bifea/5975

Torres Tovar, C. (2002). La ciudad: espacio de inclusión y exclusión. En C. A. Torres Tovar, F. Viviescas Monsalve \& E. Pérez Hernández (coords.), La ciudad: hábitat de diversidad y complejidad (pp. 318-332). Bogotá: Universidad Nacional de Colombia. https://bit. ly/2C4wUu3

Unidad Administrativa Especial de Catastro Distrital. (2017). Mapas Bogotá. https://bit. ly/2vfDR8e

Uribe, C. \& Pardo, C. (2006). La ciudad vivida: movilidad espacial y representaciones sobre la estratificación social en Bogotá. Universitas Humanistica, 62, 169-203. https://bit. ly/2RBhYZy

Urry, J. (2011) Mobilities. Cambridge: Polity Press.

Yeoh, B. \& Huang, S. (2010). Transnational domestic workers and the negotiation of mobility and work practices in Singapore's Home- Spaces. Mobilities, 5(2), 219-236. https:// doi.org/10.1080/17450101003665036 